Ellen Van ObberghenSchilling Jacques Pouysségur

\title{
Le récepteur de la thrombine et ses implications dans la prolifération des cellules vasculaires
}

Surtout connue pour son action enzymatique protéasique dans la coagulation sanguine, la thrombine exerce aussi de nombreux effets cellulaires, sur les cellules circulantes (plaquettes, leucocytes et lymphocytes $T$ ) et sur les cellules de la paroi vasculaire, initiant les processus qui suivent la coagulation et stimulent la croissance cellulaire. Elle participe ainsi à l'orchestration de la réponse inflammatoire et réparatrice qui a lieu à la suite d'une lésion de la paroi vasculaire. La thrombine exerce ses effets cellulaires par l'intermédiaire d'un récepteur appartenant à la famille des récepteurs à sept passages transmembranaires dont les messages sont relayés par le système des protéines G. Deux systèmes au moins sont couplés au récepteur de la thrombine : l'activation de la phospholipase $\mathbf{C}$ et de la protéine kinase $\mathbf{C}$ par l'intermédiaire d'une protéine $\mathbf{G q}$, et un système couplé à une protéine Gi dont l'activation gouverne l'entrée des cellules quiescentes dans le cycle cellulaire. La thrombine est un mitogène puissant des cellules musculaires lisses vasculaires et des fibroblastes. Comme la prolifération des cellules musculaires lisses vasculaires est à la base de l'athérogenèse et des complications des greffes vasculaires, la mise au point d'antagonistes bloquant le récepteur de la thrombine devrait permettre de lutter efficacement contre l'athérosclérose et les resténoses.

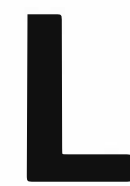

a thrombine est une protéase à sérine, engendrée par protéolyse de la prothrombine, lors d'une lésion de la paroi vasculaire. Surtout connue pour son action enzymatique dans la cascade de la coagulation sanguine, elle exerce aussi de nombreux effets cellulaires au niveau des vaisseaux (schématisés sur la figure $1)$. D'abord une action sur les cellules circulantes, en particulier sur les plaquettes dont la stimulation par la thrombine déclenche agrégation et sécrétion du contenu des granules. Les études in vitro ont montré que la thrombine est chimiotactique pour les leucocytes (monocytes et neutrophiles) 


\section{RÉFÉRENCES}

1. Carney DH. Postclotting cellular effects of thrombin mediated by interaction with high-affinity thrombin receptors. In : Berliner $\mathrm{L}$, ed. Thrombin structure and function. New York: Plenum, 1992: 351-96.

2. Ross R. The pathogenesis of atherosclerosis : a perspective for the 1990's. Nature 1993 ; $362: 801-9$.

3. McBride W, Lange RA, Hillis LD. Restenosis after successful coronary angioplasty: pathophysiology and prevention. $N$ Engl J Med 1990 ; 318 : 269-73.

4. Van Obberghen-Schilling E, Pouysségur J. Signaling pathways of the thrombin receptor. In : Zucker M, ed. Thrombosis and haemostasis 1993 State of the Art. Stuttgart : Schattauer, 1993 : 163-7.

5. L'Allemain G, Pouysségur J. L'échangeur $\mathrm{Na}^{+} / \mathrm{H}^{+}$: caractérisation et rôle physiologique. médecine/sciences $1987 ; 3$ : 382-8.

6. Vu TKH, Hung DT, Wheaton VI, Coughlin SR. Molecular cloning of a functional thrombin receptor reveals a novel proteolytic mechanism of receptor activation. Cell 1991; 64: 1057-68.

7. Rasmussen UB, Vouret-Craviari V, Jallat $\mathrm{S}$, Schlesinger $\mathrm{Y}$, Pagès $\mathrm{G}$, Pavirani $\mathrm{A}$, Lecocq JP, Pouysségur J, Van ObberghenSchilling E. cDNA cloning and expression of a hamster $\alpha$-thrombin receptor coupled to $\mathrm{Ca}^{2+}$ mobilization. FEBS Lett 1991 ; $288: 123-8$.

8. Zhong C, Hayzer DJ, Corson MA, Runge MS. Molecular cloning of the rat vascular smooth muscle thrombin receptor. J Biol Chem 1992; 267 : 16975-9.

9. Vu TKH, Wheaton VI, Hung DT, Charo IF, Coughlin SR. Domains specifying thrombin-receptor interaction. Nature 1991 ; $353: 674-7$.

10. Hung DT, Vu TKH, Wheaton VI, Charo IF, Nelken NA, Esmon N, Esmon CT, Coughlin SR. "Mirror image " antagonists of thrombin-induced platelet activation based on thrombin receptor structure. $J$ Clin Invest $1992 ; 89$ : 444-50.

11. Vouret-Craviari V, Van ObberghenSchilling E, Rasmussen UB, Pavirani A, Lecocq JP, Pouysségur J. Synthetic $\alpha$ thrombin receptor peptides activate $G$ protein-coupled signaling pathways but are unable to induce mitogenesis. Mol Biol Cell $1992 ; 3$ : 95-102.

12. Chambard JC, Paris S, L'Allemain G, Pouysségur J. Two growth factor signalling pathways in fibroblasts distinguished by pertussis toxin. Nature $1987 ; 326: 800-3$.

13. Paris S, Magnaldo I, Pouysségur J. Homologous desensitization of thrombininduced phosphoinositide breakdown in hamster lung fibroblasts. J Biol Chem 1988 ;

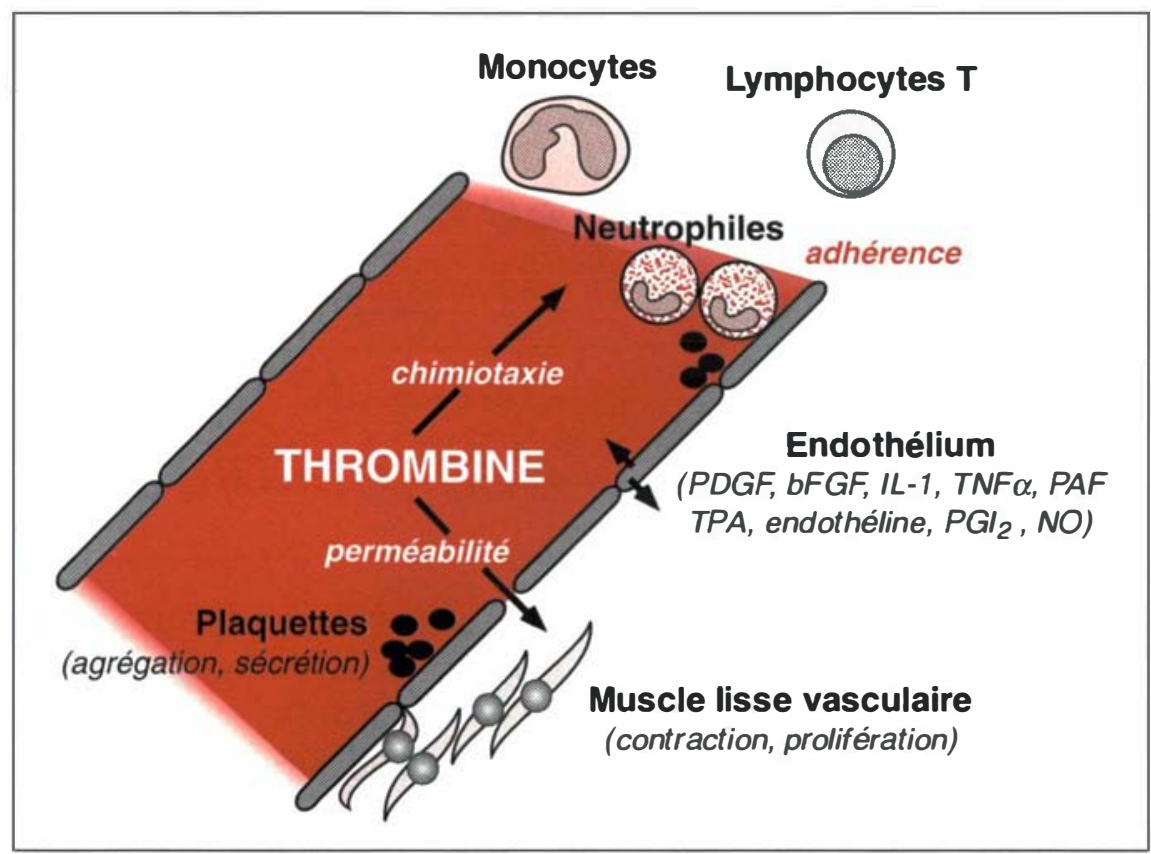

Figure 1. Représentation schématique des cibles cellulaires de la thrombine au niveau d'un vaisseau sanguin. La première cible cellulaire de la thrombine est constituée par les plaquettes dont elle provoque l'agrégation et la sécrétion des granules. Elle est chimiotactique pour les leucocytes, et induit les événements précoces associés à l'activation des lymphocytes T. Au niveau des cellules de la paroi vasculaire, elle augmente la perméabilité endothéliale, l'adhérence des leucocytes à l'endothélium, active la production de médiateurs de l'inflammation, de molécules qui contrôlent le tonus vasculaire, et de facteurs de croissance pour les fibres musculaires lisses. Lorsque I'endothélium est lésé, la thrombine agit directement sur le muscle lisse vasculaire. Abréviations: PDGF (platelet-derived growth factor), bFGF (basic FGF), IL1 (interleukine-1), TNF $\alpha$ (tumor necrosis factor $\alpha$ ), PAF (platelet activating factor), TPA (tissue plasminogen activator), $P G I_{2}$ (prostaglandine $I_{2}$ ), NO (monoxyde d'azote).

et, de plus, capable d'induire les événements précoces associés à l'activation des lymphocytes T. Enfin, la thrombine exerce des effets importants au niveau des cellules de la paroi vasculaire. Son action sur l'endothélium se traduit par: (1) une augmentation de la perméabilité vasculaire ; (2) l'adhérence de leucocytes (la première étape dans l'extravasion) en modifiant l'expression de molécules comme la P-sélectine à la surface des cellules endothéliales; et (3) la production de plusieurs substances dont : les cytokines et médiateurs de l'inflammation (Il $1, \mathrm{TNF} \alpha$, PAF), les molécules qui contrôlent le tonus vasculaire (endothéline, $\mathrm{PGI}_{2}$, $\mathrm{NO}$ ) et les facteurs qui stimulent la croissance du muscle lisse (PDGF, bFGF). Enfin, lorsque l'endothélium est lésé, la thrombine induit, par son action directe, la contraction et la prolifération des cellules du muscle lisse. L'ensemble de ces événements suggère que la thrombine participe non seulement à l'hémostase, mais aussi à l'orchestration de la réponse inflammatoire et réparatrice qui a lieu à la suite d'une lésion de la paroi vasculaire [1].

Au cours de ces dernières années, l'intérêt pour l'étude du contrôle de la division des cellules musculaires lisses vasculaires s'est considérablement accru. Il est en effet admis aujourd'hui que la réponse proliférative de ces cellules constitue un élément clé dans l'athérogenèse [2], et qu'elle joue un rôle dans les complications cliniques (en particulier resténose) survenant après angioplastie coronarienne et implantation de greffons vasculaires [3]. 


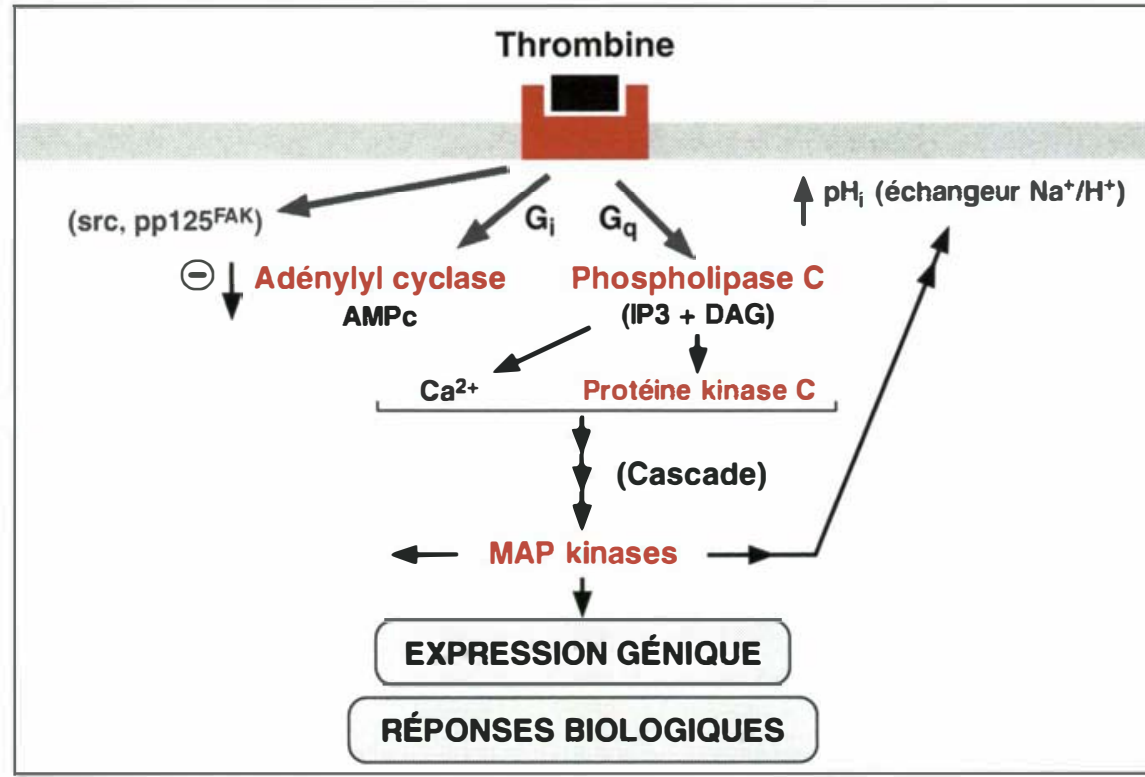

Figure 2. Voies de signalisation activées par la thrombine. La thrombine stimule au moins deux voies de signalisation intracellulaires passant par les protéines $G\left(G_{i}\right.$ et $\left.G_{q}\right)$. L'activation de ces systèmes engendre des seconds messagers, I'IP 3 (inositol-1,4,5-trisphosphate) et le DAG (diacylglycérol), impliqués respectivement dans la mobilisation intracellulaire du $\mathrm{Ca}^{2+}$ et l'activation de la PKC (protéine kinase C). La thrombine initie plusieurs cascades de kinases à spécificité sérine/thréonine, comme les MAP (mitogen activated protein) kinases, ou à spécificité tyrosine, comme des membres de la famille des src kinases ou pp 125FAK(focal adhesion kinasel. La stimulation de l'échangeur $\mathrm{Na}^{+} / \mathrm{H}^{+}$ membranaire, induisant une alcalinisation cytoplasmique, est une des multiples cibles intracellulaires activées par la thrombine et contrôlées en partie par la cascade des MAP kinases.

\section{Voies de signalisation activées par la thrombine}

Pour mieux comprendre l'action mitogène de la thrombine, il faut connaître les voies de signalisation intracellulaires activées par l'enzyme. La première étape dans l'activation d'une cellule par une hormone ou un facteur de croissance est la liaison du ligand à son récepteur spécifique. En fait, plusieurs protéines de surface fixant la thrombine ont été identifiées, comme la GPIb sur les plaquettes, la protéase nexine sur les fibroblastes ou la thrombomoduline sur les cellules endothéliales, mais le récepteur qui active les voies de signalisation intracellulaires est resté longtemps énigmatique. Néanmoins, de nombreux auteurs ont montré que la thrombine pouvait activer un grand $\mathrm{m} / \mathrm{s} \mathrm{n}^{\circ} 10 \mathrm{vol}$. 9, oclobre 93 agissent sur des protéines kinases, les réactions de phosphorylation jouant un rôle déterminant dans l'intégration des signaux et leur propagation vers le noyau, ou vers d'autres compartiments intracellulaires. Plusieurs cascades de phosphorylations, relayées par les kinases à sérine/thréonine (par exemple les MAP [mitogen activated protein kinases]) et les kinases à tyrosine (par exemple les src-like kinases) sont activées par la thrombine (pour revue voir [4]). Une des cibles de la thrombine dont l'activité est réglée par phosphorylation est l'échangeur $\mathrm{Na}^{+} / \mathrm{H}^{+}$(isoforme NHE-1), protéine membranaire ubiquitaire qui joue un rôle important dans la régulation du $\mathrm{pH}$ intracellulaire [5].

Le clonage en 1991 du récepteur de la thrombine des mégacaryocytes humains (lignée Dami) [6] et des fibroblastes de poumon de hamster (lignée CCL39) [7] a constitué une étape capitale pour l'identification moléculaire d'un récepteur dont l'existence même était très controversée, ainsi que pour l'étude des interactions de la thrombine avec les nombreux types cellulaires $\left(\mathrm{m} / \mathrm{s} n^{\circ} 5\right.$, vol. 7, p. 521). Le récepteur de la thrombine appartient à la "superfamille " des protéines à sept passages transmembranaires qui caractérisent les récepteurs couplés aux protéines G. On a trouvé $79 \%$ de similitude de séquence des acides aminés entre le récepteur humain et le récepteur de hamster, et l'analyse de l'ADN génomique par la technique de Southern suggère fortement que la protéine est codée par un seul gène [8]. Le récepteur est constitué de 427 acides aminés, avec une séquence hydrophobe située à l'extrémité $\mathrm{NH}_{2}$ du récepteur qui correspond vraisemblablement à un peptide signal (figure 3). Dans son grand domaine N-terminal extracellulaire, le récepteur possède un site de clivage spécifique par la thrombine. A côté de ce site, se trouve une séquence qui correspond au vrai ligand du récepteur, suivi par un domaine riche en résidus acides ; ce dernier présente une forte homologie de séquence avec un inhibiteur spécifique de la thrombine, l'hirudine. C'est ce domaine acide, très caractéristique des substrats de la thrombine, qui confere la spécificité de l'interaction entre la thrombine et 


\section{RÉFÉRENCES}

14. Hoxie JA, Ahuja M, Belmonte E, Pizarro S, Parton R, Brass LF. Internalization and recycling of activated thrombin receptors. J Biol Chem 1993; 268 : 13756-63.

15. Seuwen K, Kahan C, Hartmann T, Pouysségur J. Strong and persistent activation of inositol lipid breakdown induces early mitogenic events but not Go to S phase progression in hamster fibroblasts. $\mathrm{J} \mathrm{Biol} \mathrm{Chem}$ $1990 ; 265: 22292-9$

16. Seuwen K, Magnaldo I, Pouysségur J. Serotonin stimulates DNA synthesis in fibroblasts acting through $5-\mathrm{HT}_{1 \mathrm{~B}}$ receptors coupled to a $\mathrm{G}_{\mathrm{i}}$-protein. Nature $1988 ; 335$ : 254-6.

17. Herbert JM, Lamarche I, Dol F. Induction of vascular smooth muscle cell growth by selective activation of the thrombin receptor. FEBS Lett 1992 ; 301 : 155-8.

18. McNamara CA, Sarembock J, Gimple LW, Fenton II JW, Coughlin SR, Owens GK. Thrombin stimulates proliferation of cultured rat aortic smooth muscle cells by a proteolytically activated receptor. J Clin Invest 1993 ; 91 : 94-8.

19. Weiss RH, Maduri M. The mitogenic effect of thrombin in vascular smooth muscle cells is largely due to basic fibroblast growth factor. J Biol Chem 1993; 268 : 5724-7.

20. Van Obberghen-Schilling E, Chambard JC, Paris S, L'Allemain G, Pouysségur J. $\alpha$-Thrombin-induced early mitogenic signalling events and G0 to S-phase transition of fibroblasts require continual external stimulation. $E M B O J 1985 ; 4: 2927-32$

21. Weitz JI, Hudboda M, Massel D, Maraganore JM, Hirsh J. Clot-bound thrombin is protected from inhibition by heparin-antithrombin III but is susceptible to inactivation by antithrombin IIIindependent inhibitors. $J$ Clin Invest 1990 ; 86 : $385-91$

22. Bar-Shavit R, Eldor A, Vlodavsky I Binding of thrombin to subendothelial extracellular matrix. J Clin Invest $1989 ; 84$ : 1096-104.

23. Sarembock IJ, Gertz SD, Gimple LW, Owen RM, Powers ER, Roberts WC Effectiveness of recombinant desulphatohirudin in reducing restenosis after ballon angioplasty of atherosclerotic femoral arteries in rabbits. Circulation $1991 ; 84: 232-43$.

24. Nelken NA, Soifer SJ, O'Keefe J, Vu TKH, Charo IF, Coughlin SR. Thrombin receptor expression in normal and atherosclerotic human arteries. J Clin Invest 1992 ; $90: 1614-21$

25. Maraganore JM. Thrombin, thrombin inhibitors, and the arterial thrombotic process. In : Zucker M, ed. Thrombosis and haemostasis 1993 State of the Art. Stuttgart : Schattauer, 1993: 208-11.

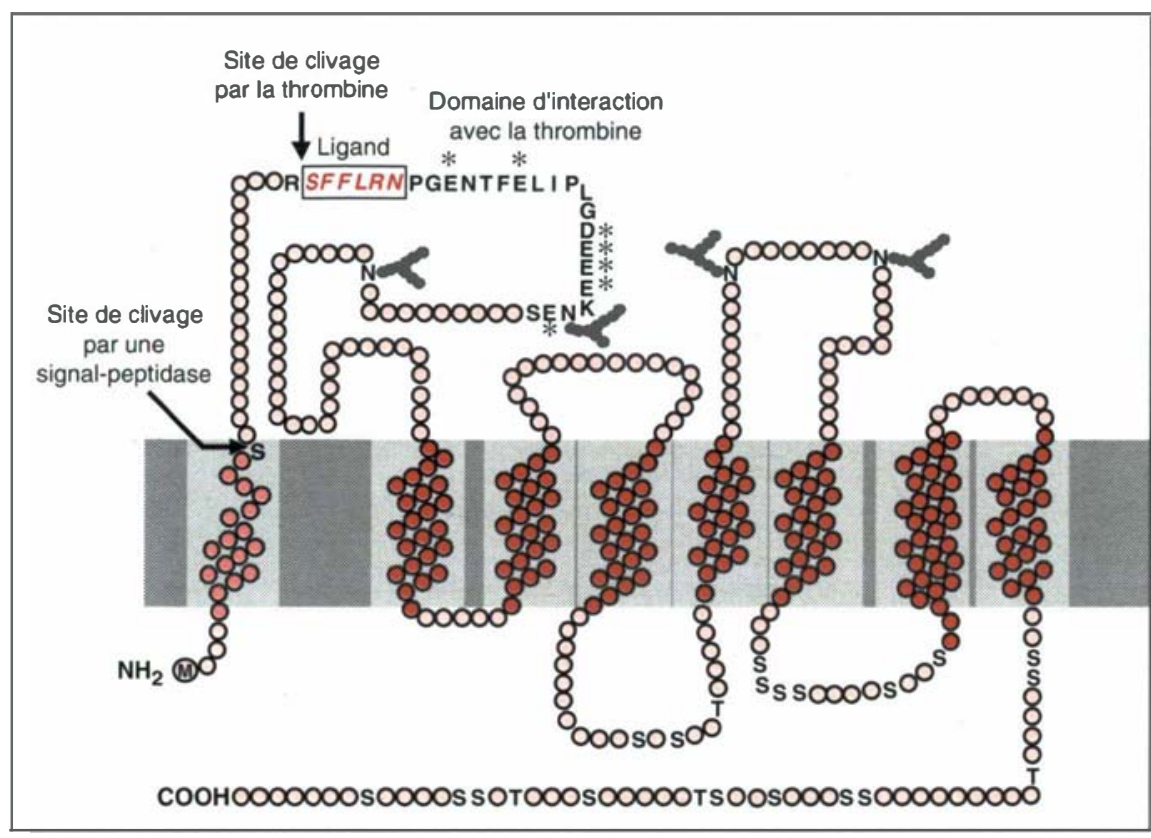

Figure 3. Représentation schématique du récepteur de la thrombine. La séquence protéique du récepteur de la thrombine obtenue à partir de l'ADNc de hamster [7] est composée de 427 résidus; pour simplifier, seuls certains acides aminés sont désignés (par leur code à une lettre). Les sites potentiels de clivage par une signal-peptidase (après la sérine 22) et par la thrombine (après l'arginine 41) sont indiqués par des flèches. La séquence qui correspond au ligand (SFFLRN) est suivie par la séquence du domaine d'interaction du récepteur avec la thrombine qui est riche en résidus acides (indiqués par des astérisques). Les sites potentiels de glycosylation (N), et de phosphorylation (S et T) sont indiqués.

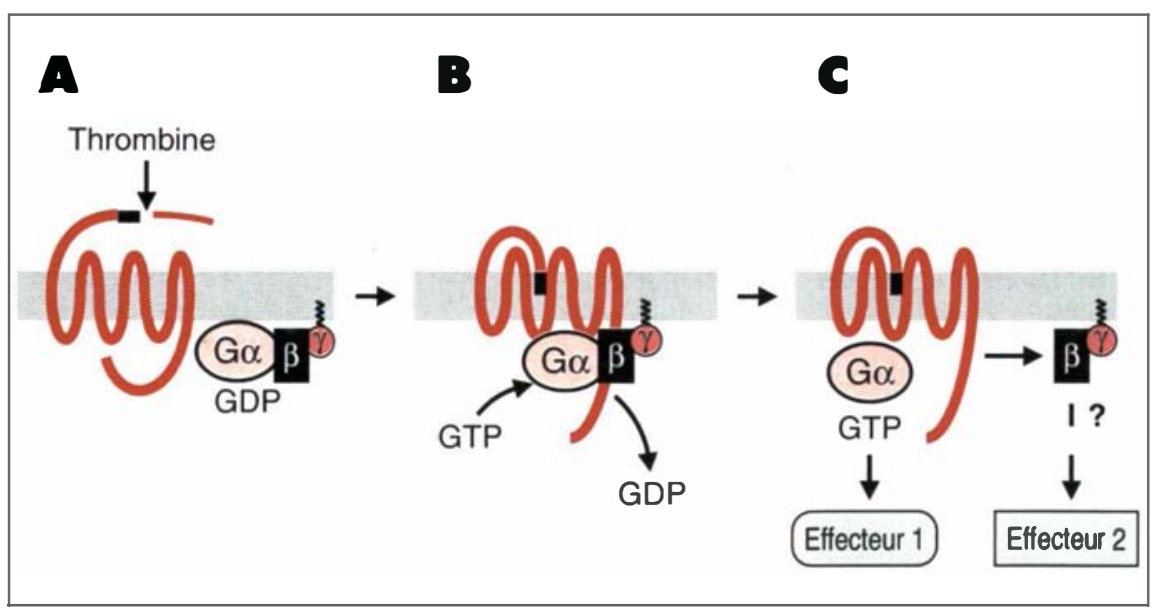

Figure 4. Modèle d'activation de protéines $G$ hétérotrimériques par le récepteur de la thrombine. (A) Activation du récepteur. Le clivage du récepteur par la thrombine démasque le ligand qui se fixe à son site de liaison sur le récepteur. (B) Échange nucléotidique. La fixation du ligand induit un changement de conformation du récepteur catalysant son interaction avec les protéines $G$. A l'état non stimulé, les protéines $G$ sont hétérotrimériques $(\alpha \beta \gamma)$, avec le GDP lié à la sous-unité $\alpha$. Le récepteur activé catalyse l'échange du GDP pour GTP au niveau de la sous-unité $\alpha$, ce qui entraîne sa dissociation du dimère $\beta \gamma$ et du récepteur. (C) Activation d'effecteurs. La forme activée $\alpha$-GTP stimule son effecteur approprié. Le complexe $\beta \gamma$ est aussi capable d'activer certains effecteurs. Dans le cas du récepteur thrombine, au moins deux protéines $G$ distinctes $\left(G_{q}\right.$ et $\left.G_{i}\right)$ sont coactivées. 
ses substrats physiologiques (fibrinogène par exemple). Le récepteur possède quatre sites potentiels de glycosylation, ainsi que plusieurs sérines et thréonines, dans les boucles intracellulaires et la partie Cterminale cytoplasmique, qui pourraient être des sites de phosphorylation intervenant dans la désensibilisation du récepteur après stimulation.

\section{Mécanisme d'activation du récepteur}

Comme l'activité protéolytique de la thrombine est nécessaire pour la quasi-totalité de ses actions biologiques (agrégation plaquettaire, mitogénicité), la présence d'un site de protéolyse par la thrombine suggérait que le récepteur pouvait être un substrat de la thrombine. En effet, les études de mutagenèse dirigée du récepteur, ou les expériences avec des peptides synthétiques correspondant au site de clivage (mais rendus non clivables) entrant en compétition avec le récepteur pour coupure par la thrombine, ont montré que le clivage après l'arginine-41 est à la fois nécessaire et suffisant pour l'activation du récepteur $[6,9,10]$. Donc, selon le modèle actuel d'activation du récepteur, la thrombine, en clivant son récepteur entre les résidus arginine-41 et sérine-42, démasque une nouvelle extrémité $\mathrm{NH}_{2}$ qui joue le rôle de ligand (figure 4). En faveur de ce mécanisme d'activation par protéolyse, il a pu être montré que des peptides synthétiques, correspondant à la séquence du récepteur à partir du nouveau résidu N-terminal (sérine-42; figure 3 ), sont capables de mimer l'action de la thrombine dans l'agrégation des plaquettes humaines [6], et l'activation des voies de signalisation couplées à des protéines $\mathrm{G}$ dans les cellules en culture [11]. Les études menées par plusieurs groupes sur la relation structureactivité des agonistes peptidiques du récepteur de la thrombine ont révélé que les 6 premiers résidus, SFFLRN* (récepteur de rongeur) ou SFLLRN* (récepteur humain), sont capables d'induire une activation maximale du récepteur (les peptides de 6 résidus

\footnotetext{
- $S=\operatorname{Ser} ; F=$ Phe; $L=$ Leu ; $R=A r g$; $N=A s n$.

sont les plus puissants). De plus, le groupement $\mathrm{NH}_{3}{ }^{+}$du résidu $\mathrm{N}$ terminal est crucial pour l'activité agoniste du peptide.

Après clivage par la thrombine, le ligand engendré se fixe sur un site du récepteur, jusqu'à présent non identifié. Cette fixation induit un changement de conformation qui permet l'interaction du récepteur avec les protéines $\mathrm{G}$ (figure $4 B$ ). A l'état basal, les protéines $\mathrm{G}$ sont sous forme hétérotrimérique $(\alpha \beta \gamma)$, avec une molécule de GDP liée avec une forte affinité à la sous-unité $\alpha$. Le récepteur activé catalyse l'échange de GDP pour GTP, ce qui a pour conséquence la séparation de la sous-unité $\alpha$ du dimère $\beta \gamma$ et sa dissociation du récepteur. Le complexe G $\alpha$-GTP est donc libre pour interagir avec son effecteur (figure $4 C$ ). Des données récentes suggèrent que les sous-unités $\alpha$ ne sont pas seules à avoir une interaction fonctionnelle avec des effecteurs, que les dimères $\beta \gamma$ peuvent aussi interagir avec un effecteur (l'isoforme $\beta 2$ de la phospholipase $\mathrm{C}$, par exemple). Cette action pourrait expliquer le fait que la stimulation d'une activité phospholipase C par la thrombine est, dans certains modèles cellulaires, partiellement sensible à la toxine de Bordetella pertussis [12]; comme nous l'avons vu plus haut, le récepteur de la thrombine est capable de stimuler au moins deux protéines $G$, une protéine de type $G_{a}$, insensible à la toxine de Bordetella pertussis, et une protéine de type $G_{i}$, sensible à l'effet " découplant ", et donc inhibiteur, de la toxine. La thrombine serait donc capable de stimuler deux isoformes de l'enzyme, l'isoforme $\beta 1$ par la sousunité $\alpha$ de la protéine $G_{q}$, et l'isoforme $\beta 2$ via le dimère $\beta \gamma$ dérivé de la protéine $G_{i}$.

La stimulation du récepteur par la thrombine, ou par les agonistes peptidiques, entraîne un état de désensibilisation qui se traduit par l'atténuation de la réponse biologique [13]. Une étude récente sur des lignées mégacaryoblastiques (HEL et CHRF-288) a mis en évidence l'internalisation très rapide du récepteur après activation [14]. Selon ces auteurs, plus des trois quarts des récepteurs de la thrombine sont transférés dans des compartiments lysosomiaux où ils sont dégradés. Le quart restant est recyclé à la surface de la membrane cellulaire, dans une conformation ne pouvant être activée par la thrombine si la stimulation a été faite par la thrombine (récepteur modifié par protéolyse), ou dans une conformation pouvant être activée dans le cas d'une stimulation par le peptide synthétique (qui n'entraîne pas le clivage du récepteur). Le renouvellement complet des récepteurs à la surface de la cellule dépend d'une synthèse de novo qui nécessite environ 20 heures.

\section{Rôle du récepteur dans la prolifération cellulaire}

Les voies de transduction de la signalisation mitogène de la thrombine, initiées par l'activation de protéines $\mathrm{G}$, varient selon la cellule examinée et son besoin en facteurs de croissance. Nos résultats, obtenus au cours de ces dernières années sur une lignée de fibroblastes, CCL39, qui possède de nombreuses caractéristiques des cellules musculaires lisses, indiquent que l'activation de la phospholipase C, bien que forte et prolongée, ne constitue pas un événement suffisant pour induire la prolifération cellulaire [15]. En revanche, l'activation concomitante d'une voie de signalisation impliquant une ou plusieurs protéines du type $G_{i}$ est cruciale pour la réponse mitogène [16]. En accord avec cette observation, la toxine de $B$. pertussis inhibe de façon importante l'effet stimulateur par la thrombine de l'entrée des cellules quiescentes dans le cycle cellulaire. Dans les cellules CCL39, les peptides synthétiques du récepteur de la thrombine ne sont pas capables de mimer la forte action mitogène observée avec la thrombine [11]. En revanche, une synergie importante pour la stimulation de la synthèse de l'ADN est observée entre les agonistes peptidiques du récepteur de la thrombine, et les facteurs de croissance dont l'action est transmise via des récepteurs à tyrosine kinase, comme le FGF (fibroblast growth factor), ou le PDGF (platelet derived growth factor). Ces études sur les fibroblastes indiquent que la puissante activité mitogène de la thrombine résulte de l'activation conjointe de plusieurs 
voies de signalisation : (1) des voies qui passent par l'intermédiaire des protéines $G, G_{i}$ en particulier, et (2) une ou plusieurs voies qui impliquent des phosphorylations sur tyrosine (relayées, soit directement par un récepteur non identifié, soit de façon indirecte par une tyrosine kinase/phosphatase). Dans les deux cas, cette action paraît dépendre de l'action protéolytique de la thrombine.

En ce qui concerne les cellules de muscle lisse vasculaire en culture, l'activation du récepteur de la thrombine par les peptides synthétiques est suffisante pour stimuler la réinitiation de la synthèse de l'ADN $[17,18]$. Comme pour les fibroblastes, la contribution d'une voie sensible à la toxine de $B$. pertussis, et donc liée à l'activation de $G_{i}$, est importante pour cet effet (F. McKenzie et E. Van Obberghen-Schilling, résultats non publiés) (figure 5). Néanmoins, une étude récente a suggéré l'implication éventuelle d'une voie tyrosine kinase dans l'activation mitogène de ces cellules. En effet, la thrombine stimule la libération du bFGF (basic $F G F$ ) par les cultures de muscle lisse aortique de rat [19], et, par conséquent, l'action mitogène de la thrombine pourrait résulter d'une synergie entre les voies couplées aux protéines
G (activées par le récepteur de la thrombine) et une voie tyrosine kinase (activée par le récepteur du bFGF). La stimulation concomitante de ces différentes voies de signalisation pourrait jouer un rôle important dans l'amplification de signaux mitogènes in vivo.

\section{Implications physiologiques et physiopathologiques du récepteur chez l'animal}

Plusieurs mécanismes permettent d'éliminer la thrombine après sa formation : sa dilution par le flux sanguin ou son bloquage par les inhibiteurs plasmatiques (antithrombine III, $\alpha_{2}$-macroglobuline, cofacteur II de l'héparine). Mais, pour exercer des effets cellulaires mitogènes in vivo, la thrombine doit être, pour plusieurs heures, présente et active sur son site d'action. En effet, les études sur les fibroblastes quiescents en culture ont montré que la thrombine, ainsi que la plupart des facteurs de croissance, doivent être présents pendant toute la phase préréplicative ( $\geqslant 8$ heures) afin de stimuler la rentrée dans le cycle cellulaire [20]. Dans ce contexte, il est tout à fait intéressant de noter que la thrombine ayant une activité protéolytique est incorporée dans le

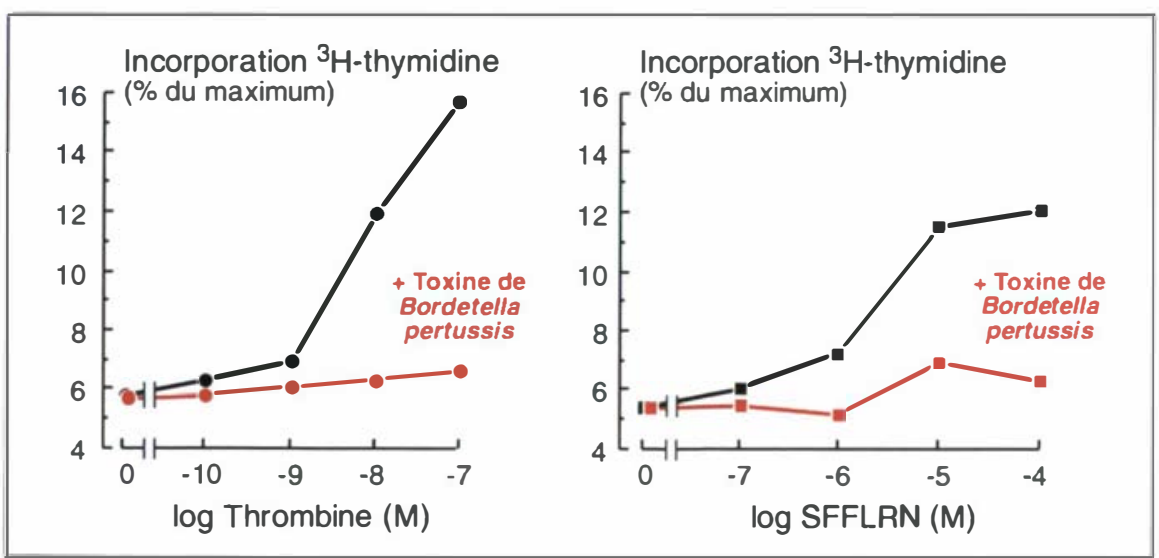

Figure 5. Stimulation de la réinitiation de la synthèse de I'ADN par la thrombine ou par I'agoniste peptidique (TRP) du récepteur. Des cellules de muscle lisse vasculaire de l'aorte de rat, rendues quiescentes par incubation pendant 48 heures en absence de sérum, ont été stimulées pendant 24 heures en présence de thymidine tritiée et de la concentration indiquée de thrombine humaine, ou d'un peptide synthétique de 7 résidus, SFFLRNP, qui correspond à la séquence du récepteur de rongeur. La présence de la toxine de B. pertussis $(100 \mathrm{ng} / \mathrm{ml})$ pendant l'incubation avec les mitogènes est indiquée. caillot de fibrine via son exosite où elle reste protégée des inhibiteurs circulants jusqu'à sa libération pendant la fibrinolyse [21]. Par ailleurs, la matrice extracellulaire des cellules endothéliales constitue également un réservoir de thrombine [22]. Il est donc tout à fait probable que la thrombine joue un rôle primordial in vivo dans la stimulation de la prolifération cellulaire. Un rôle de la thrombine a été suggéré dans la stimulation de la prolifération au niveau de l'intima dans un modèle animal de resténose après angioplastie [23]. Plus récemment, une étude a été publiée démontrant l'expression in vivo du récepteur humain de la thrombine [24]. L'expression du récepteur est faible, mais détectable au niveau de l'endothélium de la paroi de l'artère normale. Cependant, un taux élevé d'expression du récepteur de la thrombine a été observé dans les plaques athérosclérotiques humaines. Dans les lésions, l'expression est trouvée au niveau de cellules du muscle lisse vasculaire, de macrophages et de cellules mésenchymateuses non identifiées. Notre connaissance de l'action de la thrombine, relayée par un récepteur in vivo, devrait grandement bénéficier des études faisant appel au transfert ou au knock out par recombinaison homologue du gène du récepteur de la thrombine dans des modèles animaux.

Les expériences dans les modèles animaux suggèrent clairement que la thrombine joue un rôle dans la thrombose artérielle (pour revue voir [25]). En conséquence, de nombreuses équipes font des efforts considérables pour développer des antagonistes qui bloqueraient le récepteur de la thrombine au niveau des plaquettes afin d'inhiber la contribution plaquettaire à la thrombose artérielle. Mais, aussi, ces antagonistes pourraient être très utiles pour bloquer l'action mitogène du récepteur et donc restreindre la prolifération des cellules du muscle lisse à la suite d'une lésion de la paroi vasculaire, notamment dans les processus complexes conduisant à la resténose et l'athérosclérose

\section{TIRÉS A PART}

E. Van Obberghen-Schilling.

$$
m / s n^{\circ} 10 \text { vol. 9, octobre } 93
$$




\section{Summary}

The thrombin receptor and growth signaling in vascular cells

The serine protease thrombin, most widely known for its pivotal role in blood coagulation, is just beginning to be recognized as an important initiator of postclotting processes and growth stimulator. Thrombin is a potent mitogen for vascular smooth muscle (VSM) cells and fibroblasts. A great deal of attention has been directed to the proliferative response of VSM cells and its regulation, as alteration of growth is the basis of atherogenesis and clinical complications of ten observed following angioplasty and the implantation of vascular grafts. Expression cloning and sequencing of a thrombin receptor cDNA from a human megakaryocytic line and hamster fibroblasts has provided a framework to study the molecular mechanisms of thrombin action. The thrombin receptor is a member of the seven membrane-spanning receptor family that stimulates intracellular signaling pathways via $\mathrm{G}$ proteins. Thrombin activation of its receptor occurs by a unique proteolytic mechanism whereby cleavage at a defined site in the $\mathrm{N}$-terminal ectodomain unmasks a tethered ligand. Accordingly, the receptor can be fully activated by synthetic peptides ( $\geqslant$ 5 residues) corresponding to the sequence of the receptor's 'builtin' ligand. At least two $G$ protein signaling systems are functionally coupled to the receptor: (1) a Phospholipase C / Protein kinase C pathway (via a $G_{\mathrm{q}}$ protein), and (2) a $G_{i}$-coupled pathway, whose activation is essential for cell cycle re-entry. Consequently, several kinase cascades, common to those activated by receptor tyrosine kinases, are initiated by thrombin which lead to phosphorylation of key cellular components involved in signaling cell differentiation and growth. 